\title{
Compiling Path Expressions into VLSI Circuits
}

\author{
T. S. Anantharanan \\ F. M. Clarke \\ M. J. Fostert \\ B. Mishra \\ Department of Computer Science,
Carncgic-Mcllon University
Pittsburgh. Pennsylvania 15213
}

Ahstract: Path expressions were originally proposed by Campbell and Habcrmann [1] as a incchanism for process synchronization at the monitur level in software. Not unexpectedly. they also provide a useful notation for specifying the bchavior of asynchronous circuits. Motivated by this potential application we investigate how to directly translate path expressions into hardware.

Our implementation is complicated in the case of multiple path expressions by the need for synchronization on event names that are common to more than one path. Moreover, sinee events are inherently esynchronous in our mudel, all of our circuits must be self-timed.

Nevertheless. the circuits produced by our construction have area proporional to $\mathrm{N} \cdot \log (\mathrm{N})$ where $\mathrm{N}$ is the total length of the multiple path expression under consideration. 'This bound holds regardless of the number of individual paths or the degree of synchronization between paths.

\section{Int roduction}

As the boundary between software and hardware grows less and less distinci. it becomes increasingly important to investigate methods of dicctly implementing various programming language features in hardware. Since many of the problenis in interfacing hardware devices involve some form of process synchronization, language teatures for synchronization descrve considerable attention in such investigations. In this paper we consider the problem of directly implementing pith

${ }_{\text {Current address: Department of Computer Science, Columbia }}$ University, New York. New York 10027.

This research was partially supported by NSF Grant MCS-82-16706, and the Defense Aiwanced Rescarch Projects $\Lambda$ gency (DOD). $\Lambda$ RPA Order No. 3597, monitored by the Air Force Avionics Laboratury Undor Contract F33615.81-K-1539.

Permission to copy without fee all or part of this material is granted provided that the copies are not made or distributed for direct commercial advantage, the ACM copyright notice and the title of the publication and its date appear, and notice is given that copying is by permission of the Association for Computing Machinery. To copy otherwise, or to republish, requires a fee and/or specific permission.

01984 ACM 0-89791-147-4/85/001/0191 \$00.75 expressions as self-timed VLSI circuits. Path expressions were uriginally proposed by Campbell and Habermann [1] for restricting access by other processes to the procedures of a monitor. For example, the simple readers and writers problem with two reader processes and a single writer process is solved by the following multiple path expression:

$$
\begin{aligned}
& \text { path } R_{1}+W \text { end, } \\
& \text { path } R_{2}+W \text { end. }
\end{aligned}
$$

The first path expression prohibits a read operation by the first process from nccurring at the same time as a write operation. The sccond path expression enforces a similar restriction on the behavior of the second recider process. In a computation under control of the multiple path expression, the two read operations may occur simultancously, but a read and write uperation cannot occur at the same time.

Path expressions are uscful for process synchronization for two reasons: First, the close relationship between path expressions and regular expressions simplifics the task of writing and reasoning about programs which use this synchronization mechanism. Secondly, the synchronization in many concurrent programs is finite state and thus, can be adcquately described by regular expressions. For preciscly the same rcasons, path expressions arc uscful for controlling the bchavior of complicated asynchronous circuits. The readers and writers example above could equally well describe a simpic bus arbitration scheme. In fact, the finitc-state assumption may be cven morc reasonable at the hardware lcyel than at the monitor level.

Which brings us to the topic of this paper: What is the best way to translate path expressions into circuits? Iauer and Campbell have shown how to compile path expressions into Petri nets [6]. and Patil has shown how to implement Petri nets as circuits by using a PLA-like device called an asynchronous logic array [11]. Thus, an obvious method for compiling path expressions into circuits would be to first translate the path expression into a Petri net and then to implement the 
Petri net as a circuit using an asynchrouous logic airay. However. carcful examination of Laucr and Campbell's scheinc shows that a multiple path expression consisting of $M$ paths each of length $K$ can result in a Petri net with $\mathrm{K}^{\mathrm{M}}$ places. Thus, the naive approach will in general be infeasible if the number of individual paths in a multiple path expression is large.

For the case of a path expression with a single path their scheme does result in Petri net which is comparabie in size to the path expression. However, direct implementation of such a net using Patil's ideas may still result in a circuit with an unacceptably large area. An asynchronous logic array for a Petri net with P places and T transitions wi!l have at za proportional to $P$.T regardiess of the number of ares in the net. Since the nets obtained from path expressions tend to have sparse cuge sets, this quadratic behavior may waste significant chip area.

Pethaps, the work that is closest to outs is due to $1 . \mathrm{l}$ and $\mathrm{l}$.auer [8] who do indeed implement path expressions in VI.SI. However. their circuits differ significantly from ours: in particular, thcir circuits are synchronous, and synchronization with the extermal world (which is, of coursc, inherently asynchronous) is not considered. Furthermore, their circuits use PI. $\Lambda$ 's that result in an area complexity of $O\left(N^{2}\right) . \quad R e m[13]$ has investigated the use of a hicrarchically structured path expressionlike language for specifying CMOS circuits. Although he does show how certain specifications can be translated into circuits. he does not deseribe how to handle synchronization or give a general layout algorithm that produces arca cfficient circuits.

In contrast. the circuits produced by the construction described in this paper have area proportional to $N \cdot \log (N)$ where $N$ is the total length of the multiple path expression under consideration. Furthermore, this bound holds regardless of the number of individual paths or the degree of synchronization between paths. $\Lambda$ s in [3] and [4] the basic idea is to gcuerate circuits for which the underlying graph structure has a constant separator theorem [7]. For path expressions with a single path the techniques used by [3] and [4] can be adapted without great difficulty. For multiple patis with cominon event names, however, the construction is not straightforward, because of the potential necd for synchronization at many different points on cach individual path. Moreover. the actual circuits that we use must be much more complicated than the synchrouous ones used in ([3], [4]). Since events dre inherently asynchrouous in our model, all of our circuits must be self-timed. This requires the use of special circuit design techniques and significantly complicates the proof that this circuit correctly rantures the semantics of path expressions.

The paper is organized as follows: $A$ formal semantics for path expressions in terms of partially ordered multisets [12] is given in sectivn 2. In sections 3, 4, and 5 we give a hicrarchical description of our scheric for inplementing path expressions as circuits. In section 3 we first describe how the complete circuit interfaces with the external worli. We then show how to build a synchronizer that coordinates the behavior of the circuits for the individual path expressions in a inultiple path expression. In section 4 we describe a circuit for implementing single path expressions which we call a sequencer. In section 5 we show how the arbiter circuit used in section 3 can be implemented. We also argue that these circuits are correct and can be laid out efficiently. The paper concludes in section 6 with a discussion of issues such as fairness and of upen problems such as the possibility of extending our construction to other synchronization mechanisms like the oncs used in CCS and CSP.

\section{The Semantics of Path Expressions}

In this section we give a simple but formal semantics for path expressions in terms of partially ordered multiscts of events [12]. We atso relate our semantics to the one in terms of Petri Nets given by Lauer and Campbell [6].

Definition 1: A partially ordered multiset (ponsct) over $\Sigma$ is a triple $(\mathrm{Q}, \leq \mathrm{F})$ where $(\mathrm{Q}, \leq)$ is a partially ordered set and $\mathrm{F}$ is a function which maps $Q$ into $\Sigma$.

An example of a pomset is shown in Figure 2-1. We use subscripts to distinguish different instances of the same element of $\boldsymbol{\Sigma}$. Note that we could have alternatively defined a pomset as a directed acyelic graph in which each node is labcled with some element of $\boldsymbol{\Sigma}$.

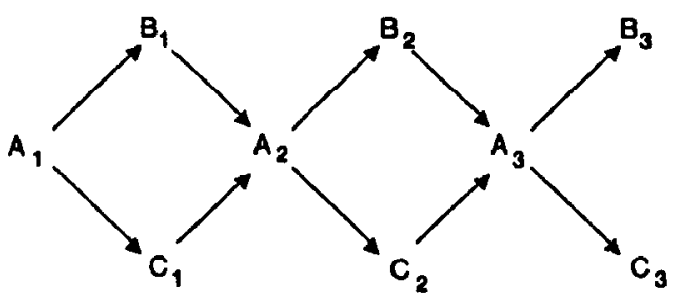

Figure 2-1: An example pomset

If the ordering relation of a poinset $P$ over $\Sigma$ is a total order, then we caln naturally associate a scquence of elements of $\Sigma$ with $P$; we will use $S\left(P^{\prime}\right)$ to denote this sequence. In fact, a pomset should be regarded as a natural generalization of a scquence in which ccrtain clements are permitted to be concurrent; this is why the concept is useful in mudeling systems where several cvents may occur simultaneously.

Definition 2: If $\mathrm{P}=(\mathrm{Q}, \leq, \mathrm{F})$ is a pomset over $\Sigma$ and $\Sigma, \subseteq \Sigma$, then the restriction of $P$ to $\Sigma_{1}$ is the pomset $\left.P\right|_{\Sigma_{1}}=\left(Q_{1} \leq_{1}\right.$. $\left.F_{1}\right)$ where $Q_{1}=\left\{d \in Q \mid F(d) \in \Sigma_{1}\right\}$ and $\leq_{1}, F_{1}$ are restrictions of $\leq, F$ to $Q_{1}$, respectively. 
If $P$ is a totally ordered pomset over $\Sigma$ and $\Sigma, \Sigma$, then $S\left(\left.P\right|_{\Sigma_{1}}\right)$ is just the subsequence of $S(P)$ obtained by deleting all of those clements of $\Sigma$ which are not in $\Sigma_{1}$.

$\Lambda$ simple parh expression is a regular expression with an outermost Kleenc star. The only operators permitted in the regular expression are (in order of precedence) "*", ";", and "+". The "*" operator is the Klcene star, ":" is the sequencing operator, and "+" represents exclusive choice. Operands are event names from some set of events $\Sigma$ that we will assume to be fixed in this paper. The outermost Kleene star is usually represented by the delimiting keyword path ... end. Thus (a) would be represented as path a end.

A multiple path expression is a sct of simplc path expressions. As we will see shortly, each additional simple path expression further constrains the order in which events can occur. However, we cannot simply take as our semantics for multiple path expressions the intersection of the languages corresponding to the individual path expressions; two events whose order is not explicitly restricted by one of the simple path expressions may be concurrent. For example, in the multiple path expression for the readers and writers problem discussed in the intreduction the two read events $R_{1}$ and $R_{2}$ may occur simultancously. Neverthcless, we will still have occasion to use ordinary regular expressions in giving the semantics for path expressions; if $R$ is an ordinary regular expression over $\Sigma$, then $\Sigma_{R} \subseteq \Sigma$ will be the set of symbols of $\Sigma$ that actually appcar in $R$ and $L_{R} \subseteq \Sigma_{R}^{*}$ will be regular language which corresponds to $R$.

Definition 3: l.ct $\Sigma$ be a finite set of events; a race over $\Sigma$ is a finite pomset $r=(Q, \leq, F)$ over $\Sigma$. We say that $i \in Q$ is an instance of an crent $c \in \Sigma$ if $F(i)=c$. $\Lambda n$ instance $i_{1}$ of event $c_{1}$ precedes an instance $i_{2}$ of event $c_{2}$ if $i_{1}$ precedes $i_{2}$ in the partial order $\leq$. An instance $i_{1}$ of event $e_{1}$ is concurrent with an instance $i_{2}$ of event $e_{2}$, if it is not the case that $i_{1}$ precedes $i_{2}$ or that $i_{2}$ precedes $i_{1}$.

In the example above $A_{1}$ precedes $A_{2}$, but $B_{1}$ and $C_{1}$ are concurrent.

Delinition 4: Let $R$ be a simple path expression with event set $\Sigma_{R}$. $\Lambda$ trace $T$ is consistent with $R$ iff $\left.T\right|_{\Sigma_{R}}$ is totally ordered and $S\left(\left.T\right|_{\Sigma_{R}}\right)$ is a prefix of some sequence in $L_{R}$. If $M$ is a multiple path expression, then a trace $T$ is consistent with $M$ iff it is consistent with each simple path expression $R$ in $M . \operatorname{Tr}_{\Sigma}(M)$ is the sct of all traces which are consistent with $M$.

Consider, for example, the multiple path expression M: pith $A ; B$ end, path $A ; C$ end.

with $\Sigma=\{\Lambda, B, C\}$. It is casy to sec that the trace in Figure $2-1$ is consistent with each of the simple path expressions in $M$ and hence is in $\operatorname{Tr}_{\Sigma}(\mathrm{M})$

\section{Synchronizers for Multiple Path Expressions}

This section describes our implementition of synchronizers for multiple path expressions. Figure 3-1 illustrates the iiterface between a synchronizer and the external world. Fach event $e$ is asseciated with a request line RTO, and acknowledge line $\mathrm{ACK}_{\dot{c}}$. The syuchronizer cooperalcs with the external world to ensure that these request and acknowledge lines follow a 4-cycle protocol:

1. The external world raises $\mathrm{RT}_{\mathrm{e}}$ to indicate that it would like to proceed with event $e$.

2. The synchronizer raises $\Lambda \mathrm{CK}$, to allow the external world to procced with cvent $e$.

3. The external world lowers RFQ, signifying completion of crent $e$.

4. The synchronizer lowcrs $A C K$, signifying the end of the cycle and permission to begin a new one.

In this implementation, an event will occur during the period between cycles 2 and 3 in this protocol, where both REQ and $A C K$ are high. Thus, multiple occurrences of any cvent $e$ are non-overlapping in time, since any two occurrences are scparated by the lowering of $\mathrm{ACK}$ and the raising of REQ.

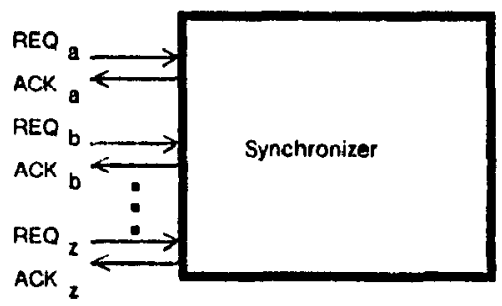

Figure 3-1: A synchronizer

An overview of a synchronizer circuit is shown in Figure 3-2. We describe below some of the building blocks in the circuit.

The $C$ gate in Figure 3.2 is a Muller C-clement; the output of a C.element remains low until all inputs are high and thereafter remains high until all inputs are low again. Its behavior then cycles. For an inplementation sec [14].

The arbiter in Figure 3-2 enforces pairwise mutual exclusion over the outputs corresponding to pairs of events which occur in the same path 


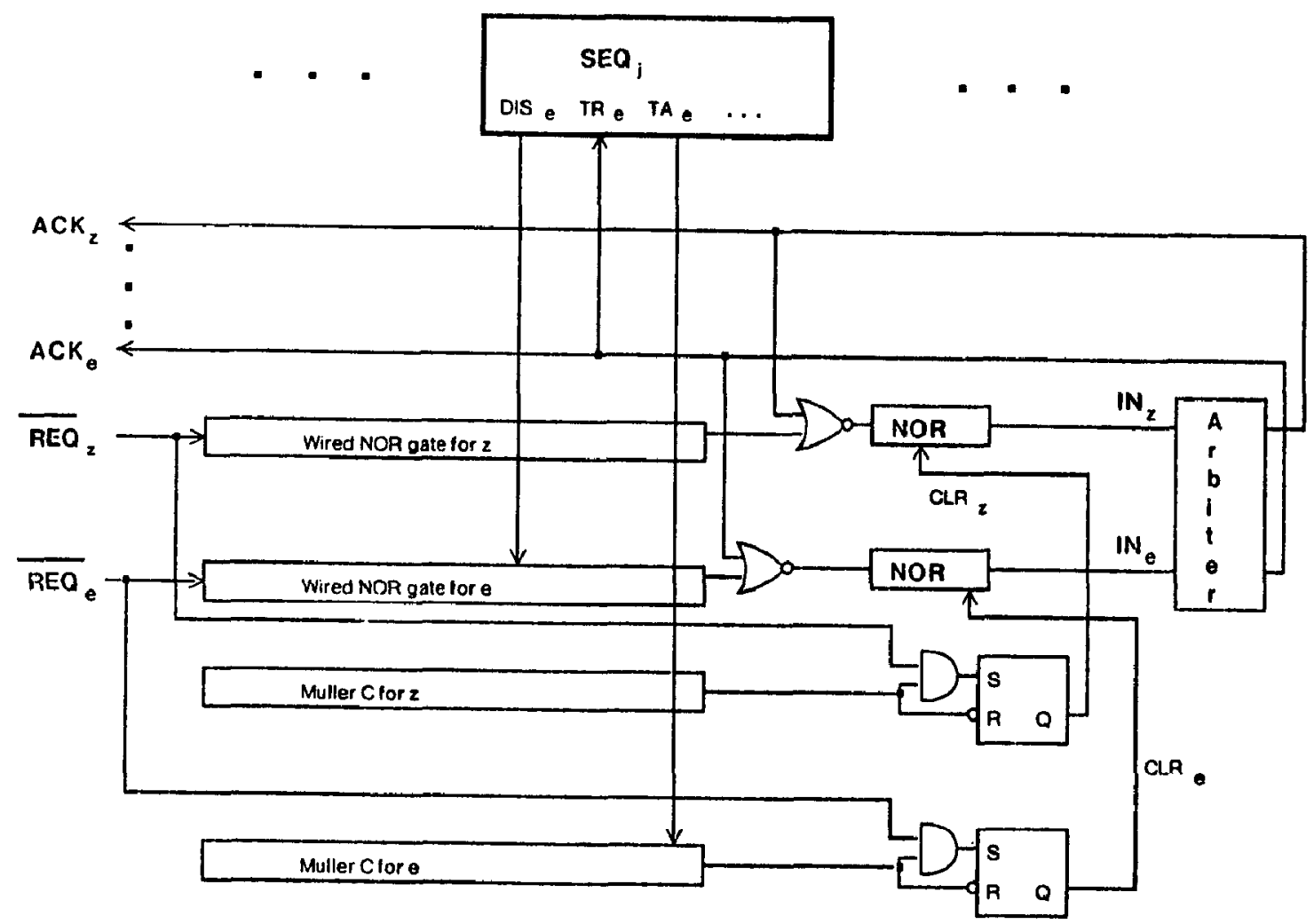

Figure 3-2: $\Lambda$ synchronizer circuit

expression.In addition to enforcing mutual exclusion the arbitcr trics to raisc any output whose input is high. Most implementations of arbiters will have metastahie states during which fewer signals than possible inay be high at the output. Despitc the metastable statcs, however, once an output signal has becn raiscd, it remains high as long as the corresponding input remains high. The implementation of such an arbiter is discussed in detail in section 5.

Fach sequencer block in Figure 3-2 ensures thit the sequence of events satisfies one of the simple path expressions that comprise the multiple path expression. The synchronizer circuit contains one sequencer for cach simple path expression. so that cacli simple path expression is satisficd by an exccution cvent trace. For each cvent $e$ that appears in a simple path, the corresponding sequencer has three connections: a request ' $T R$ ' an acknowledge $T \Lambda_{e}$, and a disable DIs $e^{\prime}$ Events are sequenced by exccuting a 4-cycle protocol over one pair of the TR/TA lines. The DIS outputs of the sequencer are only valid between these cycles (when all TR and $\mathrm{TA}$ are low), and indicate which events would violate the simple path. The synchronizer will not initiate a cycle for any cvent whose bis line is high. The implementation of the sequencer is given in section 4.

We now describe how the components of the circuit are interconnected. Refer to Higure 3-2. I.et $S F Q_{e}$ denote the set of scquencers for simple paths that contain event e. Ficry sequencer in $S E Q_{e}$ has its DIS signal connccted to a wired-NOR gate for $e_{2}$ its TA signal connected to a $\mathrm{C}$ gate for $e$, and its $\mathrm{TR}_{e}$ signal connceted to $\mathrm{ACK}_{\text {e }}$ The output of the latch at the end of the $\mathrm{C}$ gate for $e$, which is labeled CLR $e$ is connected to each of the NOR gates in front of the arbiter which corresponds to event $e$ or to some event mutually exclusive to $e$.

The following is an informal description of how the circuit works. The circuit behaves as shown in the timing diagram in Figure 3-3. When $\mathrm{RFQ}_{e}$ is raised, event $e$ is not allowed to jroceed unless each sequencer in $S E Q_{c}$ signals that at least one $e$ type transition is cnabled by ncgating $1 \mathrm{ss}_{e}$. Once this happens $\mathrm{IN}$, is raised, provided no mutually exclusive event is exceuting the second half of its cycle (and hence has its $\mathrm{Cl} . \mathrm{R}$ high). If the arbiter decides in favor of some other pending event mutually exclusive to $e$, the above process repcats until $e$ again gets a chance at the arbiter. Otherwise $\Lambda \mathrm{CK}$, will be raised and latehed by the NOR gate arrangement in front of the arbiter. At this point the external world may procecd with event $c$. Simultancuusly each scquencer in $S E Q_{e}$ will find $\mathrm{TR}_{\epsilon}$ high and after some time raise $\mathrm{TA}$, When all sequencers in $S F Q_{e}$ have raised $T_{e}$ and the external world acknowledges completion of event $e$ by lowering $\mathrm{KIQQ}_{e}$, Cl.R will be raised. This causcs $\triangle C K_{e}$ to be lowered. Fach sequencer in $S E Q_{e}$ will find $T R_{e}$ low and after some time lower $T \Lambda_{e}$. When all such sequencers are donc, CI. $R_{e}$ is lowered, and the cycic is completed. 


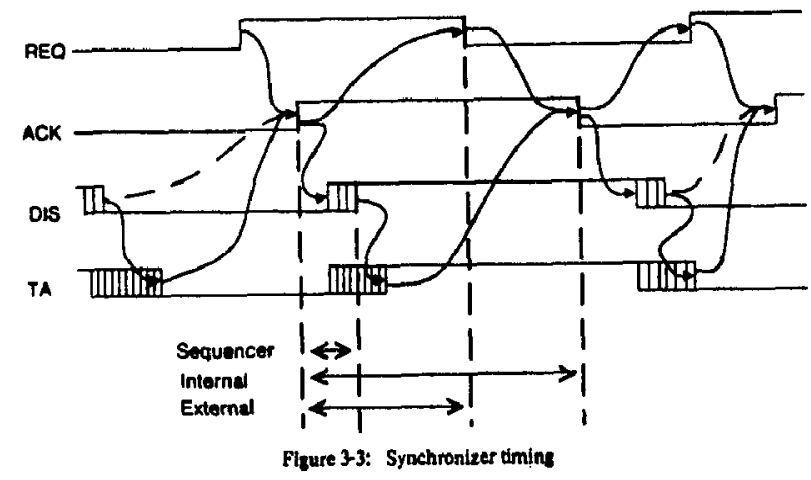

To formally establish the correctness of our circuit . we must establish two things: First, we must show that the circuit allows only semantically correct event traces; second, that the circuit will allow any semantically correct event trace for some bchavior of the extcrnal world. These properties of the circuit are often called safeness and liveness respectively. Our proof will make use of propertics of the various circuit components shown in Figure 3-2. We list the inost important of these propertics as propositions, namely those relating to the sequencer, the arbiter, and the external world. Properties of other circuit components such as SR Flip-Flops, NOR gates, etc., are assumed to be well known and are used without further discussion. The proof also makes certain assumptions about the delays of the components:

1. 'The delay of the main NOR gate plus the 2-input NOR gate is less than that of the main Muller-C element plus the $\mathbf{S R}$ Flip-Flop.

2. The maximum variation in delay for the NOR gates in front of the arbiter is less than the minimum delay of the arbiter.

We bcgin by introducing some notation that will be nceded in the proof. Let the sequencers be denoted by SI:Q ${ }_{1} \ldots$ SF $Q_{p}$ corresponding to the path expressions Rl ... Rp $\in$ M, and let $\Sigma_{\mathrm{R} 1} \ldots \Sigma_{\mathrm{Rp}}$ be the subsets of $\Sigma$ that actually appear in $R 1$... Rp respectivcly. Let I be a set of time intervals, which may include scmi-infinite intervals extending from some finitc instant to infinity. Each clement in I is labclled by an element in $\mathbf{\Sigma}$. Define $\mathrm{T}(\mathrm{I})$ to be the trace which has an element for each element in I and has the obvious partial order defined between eiements whose time intervals are non-overlapping. Referring to Figure 3-3, let

- Fxt = set of time intervals labelled 'extcrnal',

- Int = sct of time intervals labelled 'internal',

- Seq(j) = set of time intervals labelled 'sequencer' for sequencer ST:Qj.

For every interval in Int with label e there are corresponding intervals with the same label in Ext and in every Seq(j) such that $e \in \Sigma_{R j}$, namely those which start at the same time. We assume that the starting points of intervals in Int lie within some finite time period of interest, and the intervals in Ext and Seq(j) are restricted to intervals corrcsponding to those in Int.
With this notation in place we state some propositions, or axioms, that describe the propertics of the circuit of Figure 3-2. These propertics will be used to prove that the circuit is safe and live. The propositions that are not self-evident will be justificd in later sections of this paper.

Proposition 5: (Extcrnal world protocol): For all events e,

1. $R F Q$ is raiscd only if $A C K$ is low.

2. $\mathrm{REQ} \mathrm{Q}_{e}$ is lowcred only if $\Lambda \mathrm{CK}_{e}$ is high.

Proposition 6: ( 1 rbiter safety and liveness):

1. For any events el,e? that are mutually exelusive, $\wedge \mathrm{CK}_{e l}$ and $A C K$ are never high simultaneously.

2. lor any cvent $e, A C K$ is raised only if in, is raised.

3. For any crent $e_{1} \wedge \mathrm{CK}$ is lowered only if $\mathrm{IN}_{e}$ is low, and withing a of 1 , being lowered.

4. Consider a set of erents $\Sigma^{*} \subseteq \Sigma$, such that no two erents in $\Sigma$ are in the same path expression. Then if all $\mathbb{N}, e \in \Sigma$, arc raised, within a finite time all $\wedge \mathrm{CK}_{e}, e \in \Sigma^{f}$, will be raised.

Proposition 7: (Scquencer protocol): For any sequencer $S_{i} Q_{j}$,

1. $T A_{e}$ is raised only if $\mathrm{rR} e$ is high.

2. $T^{e} \mathrm{e}_{e}$ is lowered only if $\mathrm{TR}_{e}$ is low.

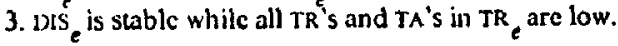

Proposition 8: (Scquencer safety and liveness) : For any sequencer $\mathrm{SHO}_{\mathrm{j}}$, assume that at all times,

- nu two TR's are high simultancously,

- TRe is raised only if DIS and all TA's are low.

- $r k_{e}^{e}$ is lowered only if $\mathrm{TA}_{e} \mathrm{e}^{\text {is high. }}$

Then the following hold:

1. $T A_{e}$ is raised within a finite time of "TR $R_{e}$ bcing raised.

2. $r{ }_{e} e_{e}$ is lowered within a finite cinc of $T R_{e}$ being lowered.

3. Fir any sequencer Sl: $Q_{i}$ whenever all TA's and TR's are low, cxactly those events $e$ will have DIs, low, for which $\mathrm{S}(\mathrm{T}(\mathrm{Ser}(\mathrm{j})))$ can be extended by $\mathrm{e}$ to give a prefix of some sequence in $\mathrm{L}_{\mathrm{Kj}}$

Proposition 9: (Initialization)

1. Sequencers are initialized with all $\mathrm{TA}$ 's low.

2. The synchronizer circuit SR flip-flops are initjalized to make all Cl.R's high.

The following theorem states that a synchronizer satisfying Propositions 5 through 9 is provably safe.

Theorem 10: (Synchronizer Safety): $T(E x t) \in \operatorname{Tr}_{\Sigma}(M)$. proof: See the appendix.

As a converse to theorem 10 we would like to show that our circuit 
can produce any valid trace Fxt, such that $l(F x t) \in \operatorname{Tr}_{2}(\mathbb{M})$ for at least some behavior of the exterinil world. However for some traces $f \in$ $\operatorname{Tr}_{\mathrm{Z}}(\mathrm{M})$, there does not exist any Fxt such that $\mathrm{T}(\mathrm{Fxt})=\mathrm{T}$. so there is no way any circuit can produce the required trace Fxt. 'This happens when $T$ does not sufficiently constrain the order in which the elements may occur so that any actual sct of time intervals will have fewer concurrent elements than $\%$. Given such a $\mathrm{T}$ it is necessary to constrain its partial order relation further, by adding additional (consistent) precedence relationships. It is casy to show using definition 4 that this will never remove $T$ from the set $\operatorname{Tr}_{2}(M)$. We shall show that whenever $T$ is sufficiently constrained so that it falls in a class of traces we call layered, then for some bchavior of the cxtcrnal world $T$ (Ext) for our circuit will equal this modified $\mathrm{T}$.

Definition 11: $\Lambda$ trace $P=(Q, 5, L)$ is called layered, if $Q$ can be subdivided into a sequence of subsets, such that for any $i l, i 2 \in \mathbf{Q}$. $i l$ precedes $i 2$ iff the subset in which $i l$ lics precedes the subsel in which $i 2$ lies.

The trace in Figure 2-1 is layered, since its elements can be subdivided into the sequence of subsets $\left\{\left(A_{1}\right),\left(B_{1}, C_{1}\right),\left(\Lambda_{2}\right),\left(B_{2}, C_{2}\right),\left(\Lambda_{3}\right),\left(B_{3}, C_{3}\right)\right\}$ with the above property. If the size of cach subsel were onc, then the trace would be totally ordered.

In gencral, any trace $P$ will have a corresponding layered trace $T$ which preserves most of the parallclism of $P$. It is casy to show that for any trace $P$, there exists a layered trace $T$, which differs from $P$ only in that the partial order relation of $P$ is a restriction of that of $T$.

Theorem 12: (Synchronizer liveness): Given any layered trace $P \in$ $\operatorname{Tr}_{\Sigma}(M)$, our circuit will producc an event trace Ext, such that $T($ Ext $)=P$ for some behavior of the external world.

proof: See the appendix.

\section{Implementing the Sequencer for a Simple Path Expression}

This section shows how to construct a sequencer that mects the conditions set forth in Propositions 7 and 8. The sequencer circuit is constructed in a syntax-dircetcd fashion based upon the structure of the simple path expression. We show that a compact layout for the sequencer exists, so that circuits of this type can be implemented economically in VISI.

Since a simple path expression is a rcgular expression, the sequencer for a simple path expression is similar to a recognizer for the regular expression. Nlthough schemes for recognition of regular languages have been proposed that avoid broadcast [3]. we will use a scheme that requires broadcast of events throughout the sequencer $[4,10]$. Because our scheme for interconnecting scquencers requircs broadcast, the broadcast within an individual sequencer carries no additional penalty. A sequencer for a simple path expression is built up from primitive cells, each corresponding to one character in the path. The syntax of the path determines the interconnection of the cells in the scquencer. In this section. we first describe the beliavior of a sequencer for a simple path expression, then give a syntax-directed construction method.

As noted in Section 3, a synchronizer communicates with each of its sequencers using three lines:

- TR; a signal to the scquencer that event $e$ is about to commence in the external world;

- TA; an acknowledgement from the scquencer that all actions started by TR, have completed;

- DIS: a status line indicating that action $e$ would violate the path constraints so that $T R_{e}$ should not be asserted.

These communication lines interact in a complex way. For a single type of event, the signals $\mathrm{TR}_{e}$ and $\mathrm{TA}_{e}$ follow the four-cycle signaling convention described in Section 3 for REQ and ACK. For differeme types of events, the synchronizer must guarantec the correct intcraction of $\mathrm{TR}$ si;nals by ensuring that only one IR signal for an crent satisfying the simple path expression is asserted at any time. The synchronizer can use the IJIS status lines to determine which requests to send to the scquencer.

The sequencer also has a part to play in ensuring the correct interaction of TK. TA and DIS. Besides gencrating a TA signal that follows the four cycic convention with $T R$, it must cnsure that the signal DIS is correct as long as no TR or TA signal is asserted. This guarantee means that if no TA is asscrted, $\mathrm{REQ}_{e l}$ and REQ $\mathrm{R}_{2}$ are both asserted, and neither DIS, nor DIS $e_{e 2}$ is truc, then the synchronizer may choose. arbitrarily between $e 1$ and $e 2$, Jetting cither of them through to the simpic path scquencer. On recciving a $T R, e$ signal, then, the sequencer must assert $\mathrm{TA}_{e}$, adjust its internal statc to rcflect the occurrence of event $e$, assert the proper set of DIS lines, and await the negation of $T R_{e}$ bcfore negating ' $T A^{\prime}$ '

Now that the behavior of a sequencer has been described, we show how to construct a scquencer for any path. $\mathrm{A}$ sequencer has two parts: a controller and a recognizer. The controller is connected diroctly to the rest of the synchronizer and generates both the TA signals and some control signals for the recognizer. The recognizer keeps track of which crents in the path have been seen and generates the DIs signals.

Figure 4-1 shows the controller for a simple path $P$. The controller accepts the signals $T R$, from the sequencer for cach event 2 that appears in P. It generates the signals $\mathrm{TA}_{e}$ along with Start $\mathrm{p}$ and End $\mathrm{p}$. The 


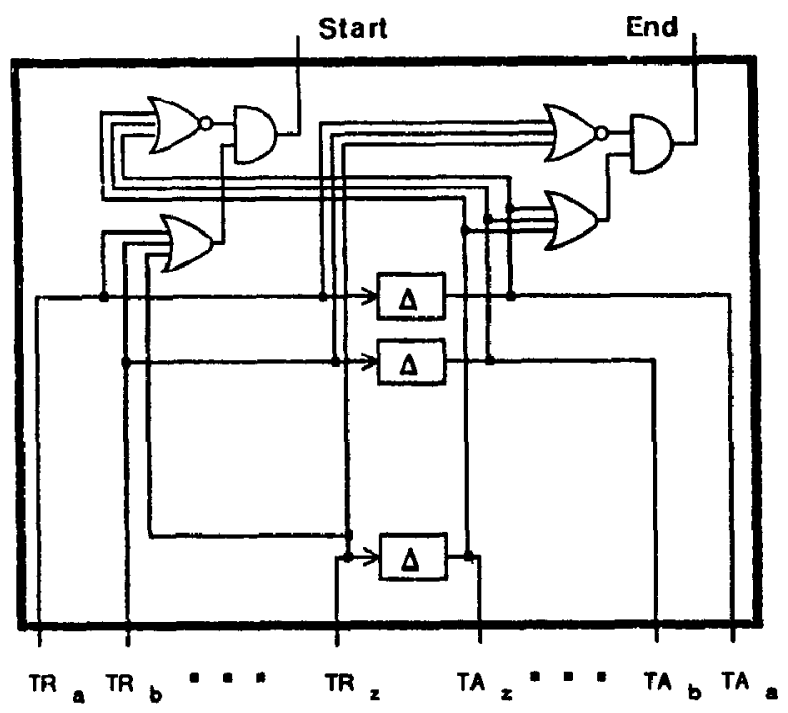

Figure 4-1: The controller for path $P$

meaning of $\mathrm{TA}_{\boldsymbol{e}}$ is that all actions caused by $\mathrm{TR}_{\boldsymbol{e}}$ have been completed. In this realization. TA is just a delayed version of TR, where the delay is long enough to let the sequencer stabilize. An upper bound on this delay can be computed from the layout of the rest of the circuit. It is possible to use a self-timed version of this circuit in which the delay is derived from the recognizer. It has been omitted in this version of the paper as it unnecessarily complicates an understanding of how the circuits work. Start $p$ and End ${ }_{p}$ are control signals that control the movement of data through the recognizer for $P$. Start $p$ is true whenever at least one TR is on and no TA is on, while End $p$ is true whenever at least onc $T A$ is on and no TR is on.

The recognizer for a path accepts the $\mathrm{rR}_{e}$ signais and generates the DIS signals. It is made up of sub-circuits corresponding to subexpressions of the path. To construct the recognizer for a path, we parse the path using a context-free grammar. Productions that are used in parsing the path determine the interconnections of sub-circuits to form the recognizer. Non-terminals that are introduced in the parse correspond to primitive cells used in the circuit.

Recognizers are constructed using the following grammar for simple path expressions.

$$
\begin{aligned}
& S \rightarrow \text { path } R \text { end } \\
& R \rightarrow R ; R|(R+R)|(R)^{*} \mid \text { <event } .
\end{aligned}
$$

The terminal symbols in the grammar correspond to primitive cells; there is one type of cell for the "+" symbol, one for the "*" symbol. onc for the ":" symbol, and one for each event. The non-terminals correspond to more complex circuits that are formed by interconnecting the primitive cclls. Using the method described in [2]. semantic rules attached to the productions of the grammar specify how the circuits on the right of each production are interconnected to form the circuit on the left.
To kecp track of which events in the path have occurred and which are lcgal, the sub-circuits of a recognizcr communicatc using the signals ENB (cnable) and Rls (result). 'The circuit for a subexpression accepts EN/3 and uses it to detcrmine when the first event in the subexpression is legal. It gencrates RES when the last event has occurred.

Figure 4-2 shows the cell for event $e$. Two latches, clocked by the signals Start $p_{p}$ and End ${ }_{p}$. control the flow of ENB and RES signals. Because of the definitions of Sturt $p$ and End $p$, the Icftmost latch is loaded from ENB whencver at least onc $T R$ is on and no $T A$ is on, while the rightmost latch is loaded to update RES whenever at least one TA is on and no TR is on. The two latches are never loaded at the same time: in fact, because TR and TA follow the four cycic signalling convention, there is a non-zero time between the end of the load signal for one latch and the start of the load signal for the other. Thus there is no combinational path through the cell.

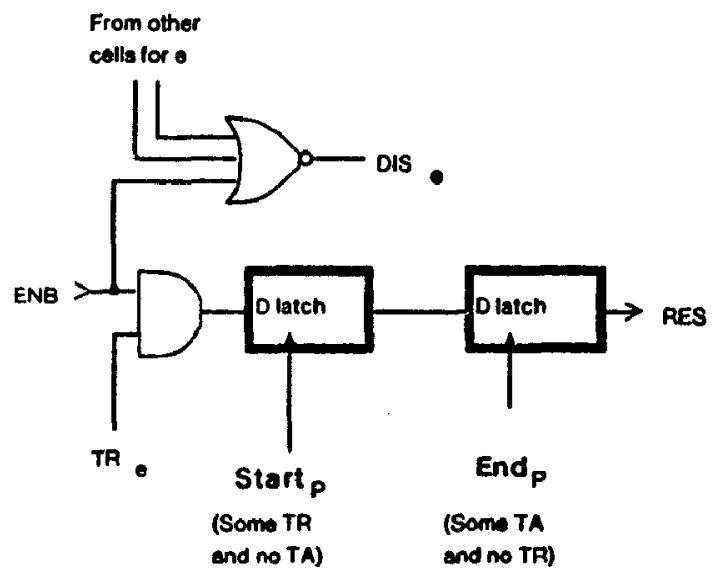

Figure 4-2: Ccll for event $e$ in path $P$

The cuent cell in Figure 4-2 propagates a 1 from I:NB to RIS only if event $e$ occurs. When this cell is used in a recognizer for a path expression, the liNh input will be true if and only if event $e$ is permitted by the expression. Thus. if $1 \mathrm{NB}$ is true it negates DIS for the path, as shown in the figurc. When a request TR is made, the output of the AND gate is loaded into the leftmost latch. If this request is $T_{e}$ this output is 1: utherwise it is 0 . In either case the output of the AND gate is piupagated to REs through the latch when TR is lowered.

Figures 4-3 and 4-4 show the cells for the ";" and "+" operators. These are strictly combinational circuits. The circuit for ";" feeds the Ris signal from the circuit at its left into the ENB signal for the circuit to its right. The circuit for "+" broadcasts its ExB signal to its operands and combines the RI:S signals from its operands in an OR gate.

Figure 4-5 shows the cell for the "*" operator. The cell enables its operand after receiving cither a 1 on either its own ENB or its operand's FIS. Every time the operand is enabled the "*" cell also puts out a 1 on 

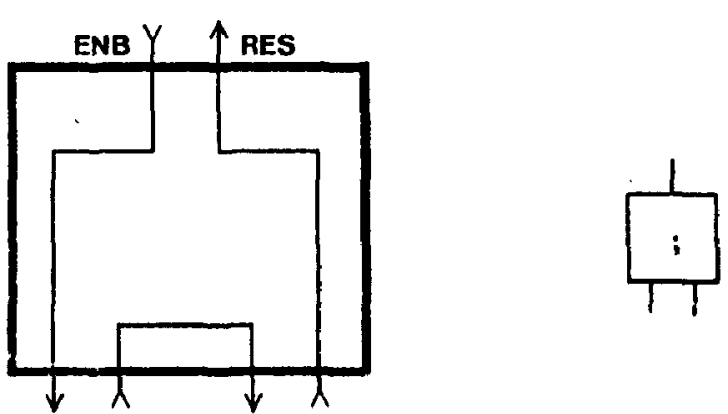

Figure 4-3: Cell for ";";

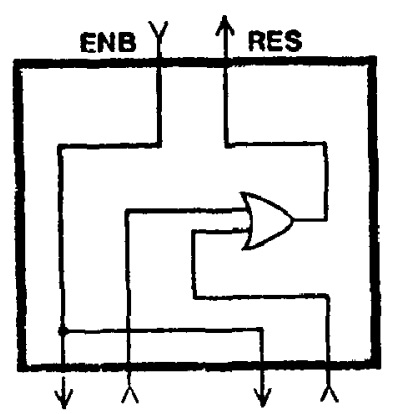

Figure 4-4: Cell for "+"

its ounn RES. It Liv. Ifforc outputs 1 on RES after 0 or more repetitions of its operand's cxpression. The additionai A.D gate sets the output to zero momentarii, anter each event, thereby preventing the formation of a laich when two or inore "*" cells are used together or when the RES output is counceted to the FNB inplit.

When larger circuits are made foun these cells, the RIS and ENB signals retain their meanings. Each event cell or sub-circuit formed from screral cclls accepts one input ENB and produces one output RES. We define ENB and RES to be correct if they meet the following conditions.

- ENB is true for a sub-circuit if cach sequence of events satisfying the expression for the sub-circuit may be the next sequence to occur.

- RES is une for a sub-circuit if some scquence of events satisfying the sub-circuit has just occurred, and FNB was true before the beginning of that sequence.

The ENB and RES signals thus indicate that a subcircuit may start recognizing events, or that it has finished. In addition, a sequencer has a signal INIT, not shown in the figures. which clears the ENB inputs to all internal celts and sets the ENiz inputs for the cells corresponding to the first crents in the path.

The semantic actions for the productions of tive gratmmar describe the interconnections of the cells in Figures 4-2,4-3 and 4-4. Attributes are attached to the symbols of the grammar to represent the sets of events that appear in the path. These sets determine which tR and TA signals are combined to produce Start $p$ and End ${ }_{p}$.

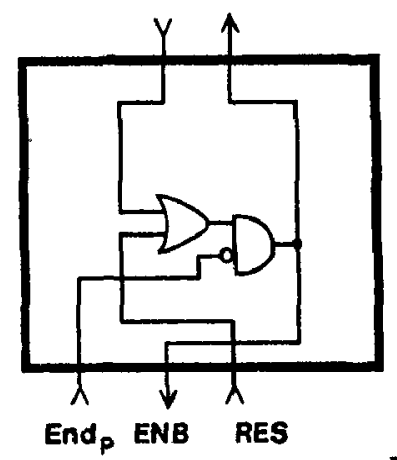

Figure 4-5: Cell for "*"*

$$
\begin{aligned}
& S[\Lambda] \rightarrow \text { path } R[\Lambda] \text { end } \\
& \text { Hook the } R E S \text { output of } R \text { to its ENB } \\
& \text { input, and connect INIT. }
\end{aligned}
$$

Figure 4-6 shows a recognizer for the path path $a ;(a+b) ; c$ end constructed using this syntax-directed technique.

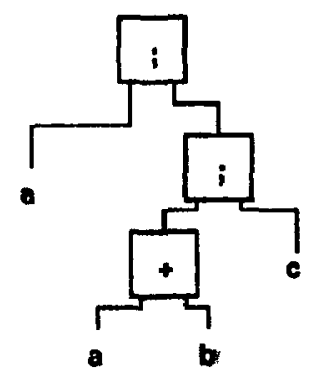

Figure 4-6: A recognizer for path $a ;(a+b) ; c$ end

All recognizers constructed by this procedure perform the corret function, as required by Propositions 7 and 8 . That is, if a recognizer is initialized and some sequence of TR signals is sent to it, the recognizer will output 1 on Dis for preciscly those events $e$ that are forbidden by the path. To prove this we show that the liNB input of an event cell in the recognizer is 1 if and only if the event corresponding to this cell is permitted by the path. $\Lambda$ s shown in Figure 4-2. DIS $e_{e} 1$ if and only if none of the cells for event $e$ is enabled. Therefure, proving that an event cell has its ENB signal set if and only if the corresponding event is permitted in the path will show that the recognizer is functionally correct. In other words, we wish to prove that all I:NB signals for event cells are correct, according to the definition of wi B above. 
We shall prove the stronger statement that all ENB signals in the recognizer are correct. This proof is based upon the structure of the recognizer. An INB signal in a recognizer is set by one of four sources:

- The operand port of a " + " or "*" cell:

- The left operand port of a ";" cell;

- The right operand port of a ";" cell;

- The INIT signal and the final RES of the recognizer;

In the first and second cases the signal is correct if and only if ENB for the operator cell is correct. In the third case the signal comes from the RES port of a recognizer for an initial subexpression. Therefore it is correct if and only if the RES signal for the subexpression is correct (asserted only at the end of the subexpression). In the fourth case the signal is correct at the start of recognition, and is correct thereafter if and only if the final RES signal is asserted only at the end of the expression. Thus, to prove that the circuits are currect, we need only prove that if the ENB signal for a recognizer is correct then so is the RES signal.

Once again, the proof of correctness is based upon the structure of a recognizer. In a correct recognizer the RES signal is true at time $t_{1}$ if and only if the ENB signal is true at some preceding time $t_{0}$ and the events between $t_{0}$ and $t_{1}$ obey the path. $A$ recognizer that is a single event cell is clearly correct. A recognizer for path $\mathbf{a} ; \mathbf{b}$ built by composition of correct subrecognizers for $a$ and $b$ is also correct, since if $\operatorname{REs}_{b}$ is true at time $t_{2}$ then there must be same time $t_{1}$ when RIS, was truc, with all intervening events satisfying path $b$. But then there must have been a time $t_{0}$ when $\mathrm{ENB}_{2}$ was true and all events between $t_{0}$ and $t_{1}$ must satisfy path a. By definition of composition. then, the events between $t_{0}$ and $t_{2}$ satisfy a;b. $\Lambda$ recognizer for path (a) ${ }^{*}$ is correct if its subrecognizer is correct, since it outputs 1 and enables its operand if and only if ENB or $\mathrm{RFS}_{2}$ is truc. Finally, a rccognizer for path $a+b$ is correct if both subrecognizers are correct, since if Ris is true then one of Rl:S or RES must be true, and if one of $\mathrm{ENB}_{\mathrm{a}}$ or $\mathrm{ENB}_{\mathrm{b}}$ is true then INB must be true. Since all methods of constructing recognizers have been shown to lead to corrcct circuits, rccognizers constructed using this procedure are functionally correct.

Now that circuits have been designed and proved correct, we give compact layouts for them. The floorplan for a sequencer, shown in Figure 4-7 has the cells that make up the recognizer arranged in a line with the controller to one side. The TR signals flow parallel to the line of recognizer cells to enter the controller, and the Start and lind signals cmerge from the controller to flow parallel to the line of cells. The ENB and RES signals that are used for intercell communication also flow parallel to the line of cells.

The laycut in Figure 4.7 is fairly small. If the sequencer for a path of

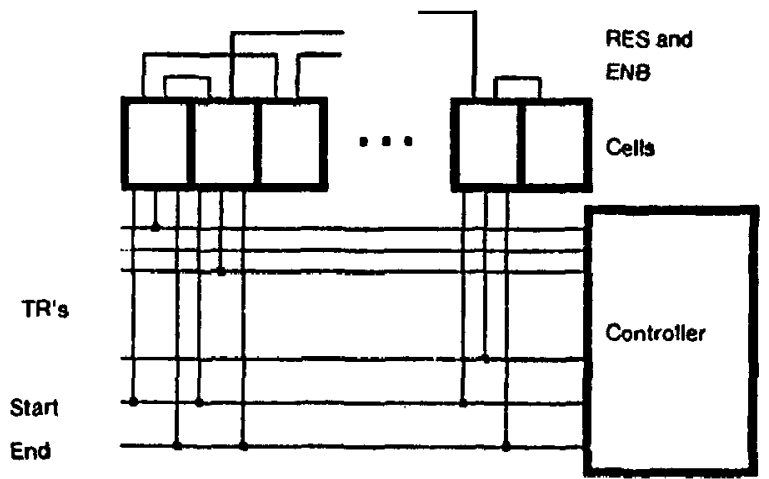

Higure 4-7: The floorplan for a sequencer

tength $n$ that has $k$ types of input events is laid out in this fashion, the arca of the layout is no more than $O(n(\log n+k))$. This is due to the structure of the recognizer circuits. All recognizer circuits are trees, which can be laid out with all nodes on a line and edges running parallel to the line using no more than $O(\log n)$ wiring tracks [7]. Thus the height of the circuit in Figure $4-7$ is $O(\log n+k)$ while its width is $\mathrm{O}(n)$.

\section{Implementation of the Arbiter}

In this section we bricfly elaborate on the arbiter shown in Figure 3-2 to show that the conditions of Proposition 6 can be met. The main function of the arbiter is to select a single cvent from a mutually exclusive set of requests. Furthermore, the arbiter must be fair - any request that remains asserted must eventually be selected.

The following observation helps to simplify the arbiter: a pair of events occurring in any single path expression must be mutually exclusive. This is duc to the role that each event plays in enforcing synchronization among a set of multiple path expressions that all contain the same named event. The arbitration function can thus be represented by a conflict graph, in which each event is denoted by a vertex and the relation between a pair of mutually exclusive events denoted by an undirected edgc. Our observation shows that the resulting conflict graph for a set of path expressions consists of a set of overlapping cliques, where a clique of $k$ nodes, $A_{1}, A_{2}, \ldots, A_{k}$ corresponds to a path expression $R$, with $\Sigma_{R}=\left\{A_{1}, A_{2}, \ldots, A_{k}\right\}$. The conflict graph represents the static structure of a set of path expressions. Figure 5-1 shows a multiple path expression with its conflict graph.

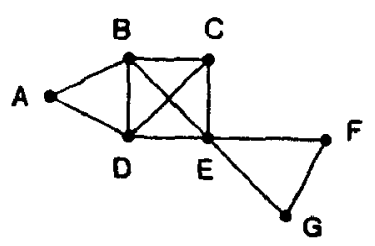

path $(A+B+D)$ end path $(B ;(C+D) ; E)$ end path $(E+F+G)$ end

Figure 5-1: The conflict graph of a path expression 
The dynamic behavior of the arhiter depends on the contlict graph togetiser with the set of events that are cnabled at any instant. The dynamic structure of the set of path expressions is represcnted by tix subgraph of the conflict graph induced by the set of vertices corresponding to the events, cnabled at that instant. The function of the arbiter is to sclect an independent sct (not neccssarily maximal) of this subgraph, thus ensuring that only one of any pair of mutually exclusive events is cnabled.

Hence an arbiter is simply a transducer that lakes a set of inputs and produces a set of outputs, subject to the constraints outlined carlier. Moren;er, it is implicitly assumed that unc arbiter is oblivious of any static or dynamic structure of the path expressions other than those represented by the conflict graph and the set of events enabled - in particular, it has no knowledge of the syntactic structure of the path expression, nor does it know the internal states of the individual scaucncers. Clearly, onc can build non-oblivious arbiters that may perform better. but this will be at the expense of conceptual simplicity and the arca nceded for additional logic and global wires.

To motivate our design we shall briefly discuss the problems with some simple schemes. In particular. we show that any deterministic obliviol:s arbiter gives rise to starvation of an event which is continually cnabled. In similar vain, we show t!at a straight-forward extension of Scity's scheme [14] for a two-input arbiter to a gencral conflict graph results in an unfair arbitcr. Finally, we present a somewhat nonstandard scheme implemented in CMOS which rectifies the problems with the other schemes.

The difficulty of building a fair deterministic arbiter can be illustrated by an example. Let $\Sigma=\left\{\Lambda_{1}, \Lambda_{2}, \ldots, \Lambda_{n}\right\}$ be a set of events. To try to build a fair arbiter for $\Sigma$ wa might assign a priority number from 0 through $n-1$ to each event. where the priority corresponds to the number of times the event is blocked. ie., the number of times the event is enabled but not selected by the arbiter. At any instant the arbiter selects from the set of enabled events with the highest priority number. When an enabled event is selected its priority number is reinitialized to the lowest value. On the other hand. if the enabled event is not selected its priority number is incremented by one. It seems that since an event $A_{i}$ can have at inost $n-1$ ncighbors in the conflict graph, and since each time it is blecked at lestst one of its neighbers is selected with a resulting increment in its own priority, after the $n^{\text {th }}$ attempt $\Lambda_{\mathrm{i}}$ must have the highest priority amons all the neighboring events and hence must be selected. Huwever, an event may never be enabled even if its request is still pending because sequencing conditions imposed by the path expression may block the event. In order to make this observation concrele consider the following path expression:

$$
\text { path }(\Lambda: C)+B:(A+B) \text { cud }
$$

Assume that the external client always rcquests permission to perform all threc cvents $\Lambda, B$ and $C$. Let the priorities of all three be 0 's initially. As a result, initially $A$ and $B$ are enabled. Assume that $B$ is selected, making B's priority 0 and A's priority 1 . In the next instant, $A$ and $B$ will again be cnabled. But now $\Lambda$ has the higher priority and will be sclected, so that $\Lambda$ 's priority becomes 0 and $B$ 's becomes 1 . Continuing in this fashion, it is casy to see that the sequence chosen will bc $B \wedge B A$ $B A \ldots$ The trouble with this scheme is that $C$ will never be enabled even if its request is pending. This example can be extended to the following lemma.

Lenuma 13: Let $M$ be a deterministic finite-state transducer inplementing an oblivious arbiter. Then there exists a path expression over $\Sigma=\{A, B, C\}$ such that one event, say $C$, will be surved cven though its request is continually pending.

Proof: Let $M$ be a deterministic finite-state transducer whose alphabet is $\Sigma=\{\mathrm{A}, \mathrm{B}, \mathrm{C}\}$. Let the states of $M$ be $S=\left\{s_{1}, s_{2}, \ldots, s_{m}\right\}$. Let the conflict graph, $G$, for the path expression be the complete graph on the vertices $A, B$ and $C$. We construct a path expression $P$ with the conflict graph $G$ such that $M$ causes the starvation of the event $C$. Notice that because of the nature ol the conflict graph $G$, if at any instant $\Lambda$ and 13 are cnabled then at most one of $\Lambda$ and $B$ may be selected by $M$.

Let $s_{1}$ be an arbitrarily chosen state of $M$. We conduct an experiment on $M$ by continuously providing $A$ and $B$ as the enabled inputs, starting with $M$ in the state $s_{1}$. If we present a string of inputs $\{A, B\},\{A, B\}, \ldots,\{A, B\}$ of length $m$ then we notice that at the $1^{\text {st }}$ input $\{A, B\}$, the transducer deterministically goes from the state $s(1)=s_{1}$ to a state $s(2)$ while outputting $A$ or $B$. Let $s(1), s(2), \ldots, s(m+1)$ be the sequence of states and $\sigma \in\{A, B\}^{m}$ be the output string produced as a result of the experiment. As a conscquence of the pigcon-hole principle, some two states in the scquence of states will be the same. Of all such pairs, let $s(i)$ and $s(j)$ be two such states closest to $s_{1}$. Assume that $i<j$ and let $k$ be the smallest multiple of $(j-i)$ such that $k \geq i$. Without luss of generality assume that $M$ outputs $B$ when in state $s(i)$ with the input $\{A, B\}$.

I.ct $P$ be the path expression

$$
\text { path }(A+B)^{r-1} ;(A ; C+B) ;(A+B)^{k-1} \text { end }
$$

It is easy to see that $P$ has $G$ as the conflict graph and if the requests for $A, B$ and $C$ are continuously pending then the scquence of outputs will be a string in $\{A, B\}^{\omega}$ and $C$ will never be cnabled. 
Before proceeding further, let us consider the path expression path $\mathbf{A}+$ $B$ end. where the conflict graph is $G=(V, E)=(\{A, B\},\{[A, B]\})$. Seit. [14] has shown how to build an arbiter for such a structure using an interlock-element, as shown in Figure 5-2.

Circuit operation in Figure 5-2 is most casily visualized starting with neither client requesting, $v_{1}$ and $v_{2}$ both near 0 volts, and both outputs high. If any single input, say $A_{\text {in }}$, is lowered then $v_{1}$ is driven bigh,

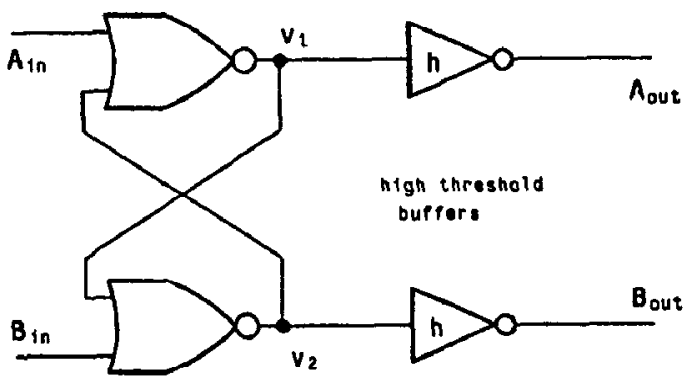

Figure 5-2: Seitz's Interlock Element

resulting in $A_{\text {out }}$ being lowered - $B_{\text {out }}$ remains unaffected. Moreover, once $\Lambda_{\text {out }}$ is lowered, and as long as $\Lambda_{\text {in }}$ is kept low, the interlock element remains in this stable state ir respective of what happens to $B_{\text {in }}$. If $\Lambda_{\text {in }}$ is now raised high, then the element returns to its initial condition it $B_{\text {in }}$ is still high; or $B_{\text {out }}$ is lowered if $B_{\text {in }}$ is lowered in the meantime.

However, the intercsting situation occurs when both $A_{\text {in }}$ and $B_{\text {in }}$ are both lowered concurrently or within a very short interval of time. In this case the cross-coupled NOR gates cnter a mctastable state, which is resolved after indeterminate period of time in favor of either $A$ or B. Since this resolution depends on the thermal noise generated by the gates, it is inherently probabilistic. In this case the outputs of the NOR gates themsclves cannot be used as the outputs. High threshold inverters between the NOR gates and the outputs prevent false outputs during the metastable condition.

It would secm natural to extend Scitz's idea by gencralizing it to the conflict graph for an arbitrary sct of path expressions. Roughly spcaking, we rnay construct a circuit by honomorphically transforming the conflict graph to a circuit by replacing each vertex with a NOR gate and cach edge with a cross-coupling of NOR gates corresponding to the pair of vertices on which the edge is incident. However, such an implementation in NMOS has some severe problenis, which will be clarified if we consider the circiit for the readers writers path expression:

$$
\begin{aligned}
& \text { path } R_{1}+W \text { end } \\
& \text { path } R_{2}+W \text { end }
\end{aligned}
$$

where the pair $R_{1}$ and $W$ and the pair $R_{2}$ and $W$ are mutually exclusive.
The conflict graph and the circuit for this expression are shown in Figure 5-3.

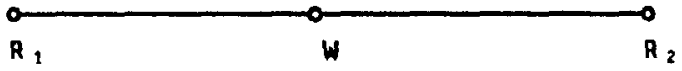

(a)

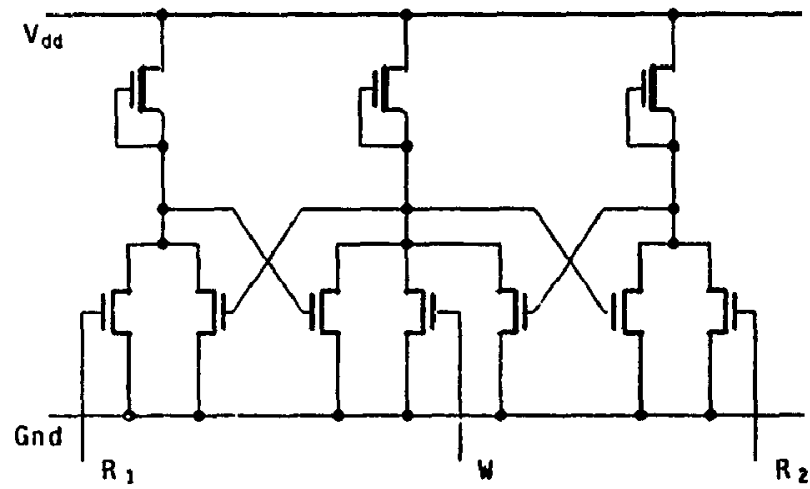

(b)

Figure 5-3: (a) The Conflict Graph and (b) The Arbiter in NMOS.

Consider the situation when the circuit is in the none-requesting conditiois and all three requests, $R_{1}, R_{2}$ and $W$. arrive concurrently. $\Lambda n$ infinitesimally shot interval $\Delta t$ after all three requests arrive, let us assume that the voltages at the outputs (of the NOR gates) have increased by an infinitesimally small value $\Delta v \ll v_{\text {th }}$. The pull-down MOS transistors may be assumed to be operating in their lincar region. Il all pull-ups arc assumed to provide cqual active resistance. the output of the NOR gate corresponding to $W$ will grow less rapidly than those corresponding to $R_{1}$ or $R_{2}$. The cumulative effect of this imbalance will result in a low output for W's NOR gate and high outputs for $R_{1}$ 's and $R_{2}$ 's. Hence if $R_{1}, R_{2}$ and $W$ request continuously then the request for $W$ will never go through, resulting in W's starvation.

An casy fix to this problem may be to increase the ratio of pull-up to pull-down for W's NOR gatc to twice that of $R_{1}$ 's and $R_{2}$ 's. But if this is done in a static manner then, when only $R_{1}$ and $W$ are requesting, $W$ will have an unfair advantage over $R_{1}$. Obviously, what is needed is some means of controlling the ratios such that depending on the set of requests the circuit configures itsclf dynamically in order to behave in a balanced fashion.

An arbitcr that can configurc itsclf dynamically for the problem with two readers and one writer is shown in Figure 5-4. To see how this scheme remedics the problem discussed earlier, consider the situation when the circuit is in non-requesting condition and all three requests, $R_{1}, R_{2}$ and $W$, arrive concurrently. An infinitesimally short interval $\Delta t$ 


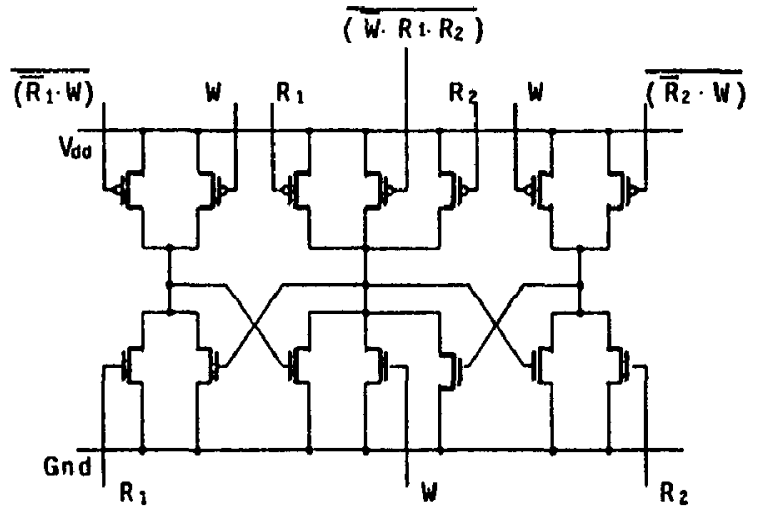

Figure 5-4: The Arbiter for 1-Writer-2-Readers Problem in CMOS.

affer all three requess arrive. the voltages at the enutpits will have increased by an infinitcsimelly small value $\Delta v$ o vo. The pull-down MOS transisturs are in their linsir region. However. 'since active resistances of the pull-up transistors depend on the neighboring events that are crabled, the pull-up resistance of the gate associated with $W$ is cxactly half of that asscuiatcd with $R_{1}$ or $R_{2}$. This provides a balance among pull-up resistances and results in almost cqual rate of growtl of voliages at the outputs. Hence une interlock elcinents enter their metastable statcs more or less simulancously; and the metastable condition is resolved cither in favour of $R_{1}$ and $R_{2}$ or in favour of $W$, the choice governed by stitistical thermal phenomena.

A similar analysis shows that the circuit behaves correctly when only two out of three requests arrive concurrently. However, if only one request, say $\mathrm{W}$, arrives while all its neighbours remain in their nonrequesting condition the circuit behaves somewhat differently. In this case the pull-up transistor with input $\overline{\left(\overline{\bar{h}} \cdot R_{1} \cdot R_{2}\right.}$ ) will turn on, thus allowing the cutput of the gate to go high. It is important to obscrve that the pull-up transistors are controlled dynamically by the requests for the neighbuuring crents - if there is a request for the neightbouring erent then only the pull-up corresponding to the event turns on: and if these is no request fur the ncighbouring events then only the pull-np corresponding to the event itself turns on. For this to be implemented criectly it is cssential that the pull-up coiresponding to the crers itscif he turned on only afuer a delay necessary for the requests for the ncigh:bouring events to propagate to the gate of the pull-up.

The cumplex statistical nature of thermal noise in the cirruit in coniunction with the complexity of the strusture of the conflict graph makes it hard io analyzc the circuit electically. For instance, the time cunstants asinciated with each arhiscr output could possibly differ significantly. Uncier the assimption that these second order effects are small, cvery cnabled event will have a positiz nun-zeris probability of being selected. Thus. for a reasunable class of pith expressions, the circuit ensures that a continuously reçüsting event is eventually' selected. 'This class includes the path expressions for which the other two arbiters can not provide a good solution.

\section{Conclusion}

So far we have not discussed fairncss. Intuitivcly, the implementation of a path expression is fair if any continuously requesting crent will be eventually selected, provided it is possible to do so without violating the semantics of the path expression. As pointed out in the previous scction, our implementation is fair for a rcasonable class of path expressions. As an example of a path expression for which our implementation is not fair consider the following :

$$
\begin{aligned}
& \text { path }(A+B) ; C \text { end, } \\
& \text { path } D ;(A+E) \text { end }
\end{aligned}
$$

Suppose that cach event taxcs the same amount of time to exceute externally and that new requests for each cvent are forthcoming as soon as allowed by the protocol. Then simultancous execution of D and $B$ will aircrnate with simultaneous execution of $\mathrm{C}$ and $\mathrm{E}$ without the arbiter cver having to block any event. Yet, cvent $A$ will never execute even if it remains continually ready. If, however, the first request for event $B$ is delayed by the time it takes to execute an event, then initial execution of event $D$ will be followed by alternate executions of $A$ and (D.C). Now $B$ and $E$ never exccute! Since neither the duration of external cvents nor the occurrence of extcrnal requests is under the control of the circuit, it is not easy to ensure fairness for such path expressions. It remains an open question whether a practical solution to this problem exists.

Since our circuits have the constant scparator property, a more compact $O(N)$ layout is be possible using the techniques of [4]. However, while it is definitely possible to automatically generate the $O(N \cdot \log (N))$ layout that we propose, it is much more difficult in practice to generate the $O(N)$ layout of [4]. Furthememe. $t_{.+} O(N)$ layout will occupy less area only for very large N. We suspect that ease of generating the layout will win over asymptotic compactness in this case.

Finally, we plan to investigate extensions of our construction to appropriate finite state subsets of CSP [5] and CCS [9]. In the case of CSP the subset will only permit boolean valued variables and messages which are signals. If the number of message types is fixed, we conjecture that area bounds comparable to those in section 4 can be obtaincd. Arrays of processes in which the connectivity of the communication graph is low can be treated specially for a more compact layout. Such a finite-state subset of CSP may even be more useful than the path expression language discussed in the paper for high level description of various asynchronous circuits. 


\section{References}

1. Campbell, R. H. and ᄉ. N. Habermann. The Specification of Process Synchronization by Path Expressions. In Lecture Noles in Computer Science. Volume 16, G. Goos and J. Hartmanis, Ed.,SpringerVerlag, 1974, pp. 89-102.

2. Foster, M. J. Specialized Silicon Compilers for Language Recognition. Ph.D. Th., CMU, July 1984.

3. Foster. M. J, and Kung, H. T. "Recognize Regular Languages with Programmable Building-Blocks." Journal of Digital Systems VI, 4 (Winter 1982), 323-332.

4. Floyd, R. W. and Ullman, J. D. "The Compilation of Regular Expressions into Intcgratcd Circuits." Journal of the Association for Computing Machinery 29, 3 (July 1982), 603-622.

5. Hoare, C. A. R. "Communicating Sequential Processes." Comm. ACM 21, 8(1978).

6. Lauer, P. E. and Campbell, R. H. "Format Semantics of a Class of High-Level Primitives for Coordinating Concurrent Processes." Acta Infornarica 5 (Junc 5 1974), 297-332.

7. Leiserson, C.E. Arca-Efficient VLSI Computation. $\mathrm{Ph}, \mathrm{D}$. Th., Carncgic-Mellon University, 1981.

8. Li, W. and P. E. I auer. A VLSI Implementation of Cosy. Tech. Rept. ASM/121, Computing Laboratory, The University of Newcastle Upon Tyne, January, 1984.

9. Milner, Robin. A Calculus of Communicating Systems. Volume 92: Lecture Notes in Computer Science. Springer-Verlag, Bcrlin Hcidelberg NY. 1980.

10. Mukhopadhyay, A. "Hardware Algorithms for Nonnumeric Computation." IlitE Transactions on Computers C-28, 6 (June 1979), 384-394.

11. Patil, Suhas $S . \wedge n \Lambda$ synchronous I.ogic $\Lambda$ rray. $M \wedge C$ 'THCHINICAI. MEMORANDUM 62, Massachuscts Institute of Technology, May, 1975.

12. Pratt. V. R. On the Composition of Processes. Symposium on Principles of Programming Languages. ACM, January, 1982.

13. Rem, Martin. Partially ordered compufations, wilh applications 10 VLSI design. Eindhoven University of ' Technology, 1983.

14. Scitz, C. L. "Ideas $\Lambda$ bout $\Lambda$ rbitcrs." L $A M B D A$ First Quarter (1980), 10-14.

\section{Appendix : Proof details}

Refer to section 3:

Lemma 14: If the same assumptions as in proposition 8 are sitisfied, then $T(S e q(j))$ is consistent with $R_{j}$.

Proof: From proposition 8 it follows that Seq(j) consists of non concurrent time intervals. The result is therefore casy to prove by induction on the number intervals in Seq(j), using the same proposition.
Lemma 15: For each element $i$ in Int with label $e_{\text {, the }}$ corresponding elements in Ext and Seq(j) are subintervals of $i$.

Proof: (requires proof based on the properties of the circuit in fig 3-2).

I.cmma 16: For any $R j \in M,\left.T(I n t)\right|_{\Sigma_{R j}}$ is a totally ordered multiset.

Proof: It is easy to show that $\left.T($ Int $)\right|_{\Sigma_{R j}}=T\left(\left.\operatorname{Int}\right|_{\Sigma_{R j}}\right)$. But Int $\left.\right|_{\Sigma_{R j}}$ consists of 'internal events' of the path expression IRj, during each of which the curresponding ACK is high. Hence by proposition 6 , no two such events overlap, and therefore $\left.T(I n t)\right|_{\Sigma_{R j}}$ is a totally ordered multiset.

Lemma 17: For any $R j \in M,\left.T(\operatorname{lnt})\right|_{\Sigma_{R_{j}}}=\left.T(E x t)\right|_{\Sigma_{R j}}$.

Proof: For any element $i$ of 'I(Int), that is also in 'T(Int) $\left.\right|_{\Sigma_{R j}}$, the corresponding clement of $T(k x t)$ will be in $|(k x t)|_{\Sigma_{R j}}$ (definition 2) since they must map to the same alphabet $e \in \Sigma_{R_{j}}$. Hence these traces have ule same number of clements. Niso from lcmuna 15 it follows that if $i /$ and $i 2$ are two elements of T(Int) $\left.\right|_{\Sigma_{R j}}$ satisfying one or none of " $i$ precedes $i 2$ " and " $i 2$ precedes $i l$ ", the corresponding clements of $\left.T(E x t)\right|_{\Sigma_{R j}}$ will satisfy at lcast the same relationships. In other words the partial order of ' $\mathrm{T}$ (Int) is a restriction of that of $T(\mathrm{Ext})$. But by $\left.1 \mathrm{cmma} 16 \mathrm{~T}(\mathrm{Int})\right|_{\Sigma_{R j}}$ is a totally ordered multiset. Hence from the above $\left.T(E x t)\right|_{\Sigma_{R j}}$ will have the same partial order relationship and, therefore, be the same totally ordered multiset.

Lemma 18: For any $R j \in M, T(S e q(j))=\left.T(I n t)\right|_{\Sigma_{R j}}$.

Proof: Follows from lemma 15 and 16 in the same way as in the proof of lemma 17. The only difference is that $\left.T(\operatorname{Seq}(j))\right|_{\Sigma_{R j}}=$ $T(\operatorname{Seq}(j))$.

Lemma 19: For any sequencer $S^{2} Q_{j}$, no two TR's are high simultaneously.

Proof: The two rR's would be two ACK's of events in the same path expression $\mathrm{Rj}$, which cannot be high simultancously by proposition 6.

Lemma 20: For any sequencer $S E Q_{j}, T_{e}$ is raised only if DIS is low and all TA's are low.

Proof: $B y$ induction on the number of rising transitions of TR's :

1. (First transition): Let the corresponding event be $e$. By proposition 9 initially all TA's are low, and all CLR's are high, hence all TK's are low initially. By proposition 7 all $T A$ 's will remain low until the first rising transition of $T R_{e}$. By the same proposition DIS, will not change until the first 
rising transition of TR. If DIS were not low, IN, would remain low (sec Figure 3-2). Hence by proposition 6, TR, would remain low, a contradiction.

2. (For a succeeding transition); let the corresponding event be $p$ and that of the previous transition $q$. While $\operatorname{TR}_{q}$ is high no $T \Lambda$ or $T R$ other than $T \Lambda_{q}$ or $T R$ can be high (proposition 6 and icmma 19). Until $\mathrm{Cl}, \mathrm{R}$ goes high, TR inust remain high (sce Figurc 3-2). Once ci $\mathrm{R}_{q}$ goes high, all $\mathrm{IN}$, with $a \in$ $\Sigma_{\mathrm{Rj}}$, will be low after a short delay (sce ligure 3-2). $\Lambda$ ssuming the variation in this delay for different $a$ 's is less than the delay of the arbiter in lowering $T R_{q}$, all TR ${ }_{a}$ with $a$ - $q$ will continue to remain low until Cl.R is lowered (sce Figure 3-2). All TA$A_{a}$, with $a=q$, aiso continue to remain low (proposition 7). But $\mathrm{Cl} . \mathrm{R}_{\text {gemains high at least until } \mathrm{TA}}$ is luwered (sec Figure 7). Hence by the time $T R$ is raised all $T \wedge$ 's will be low. Also TR could not have becn raised if IN were low (proposition 6). But if DIS was high when $\mathrm{TA}_{p}^{p}$ was last lowered then ix would now be low (sec Figure 3-2). assuming the main ROR gate plus the 2-input NOR gate have a lesser delay than the Muller-C element plus the SR Flip-Flop. Moreover, DIS cannot change before $\mathrm{TR}_{p}$ is raised (proposition 7). Hence ${ }^{p} S_{p}$ must be low when $\mathrm{TR}_{p}^{p}$ is raised.

$\square$

Lemma 21: For any sequencer $S E Q_{j}, T R_{e}$ is luwered only if $T A_{e}$ is high.

Proof: The NOR gatc arrangement in front of the arbitcr insures that once $T_{e}$ is high it remains high until $\mathrm{CLR}_{e}$ is raised, and this can occur only if TA $e$ is high (sce Figure 3-2). Morcover once TA $e$ high it will remain high until $\mathrm{TR}_{e}$ is lowered (proposition 7).

\section{Theorem 10}

Proof: l.cinmas 19,20,21 satisfy the preconditions of propusition 8 . Hence $T(\operatorname{Seq}(j))$ is consistent with $R j$ for any $R j \in M$. By lemma 18 and definition $4, T(I n t)$ is consistent with $R j$ for any $R j \in M . B y$ lemma 17 and definition $4, T(E x t)$ is consistent with $R j$ for any $R j$ $\in \mathrm{M}$. Hence by definition $4, \mathrm{~T}(\mathrm{Ext}) \in \operatorname{Tr}_{\mathbf{Z}}(\mathrm{M})$.

Lemma 22: If $T \in \operatorname{Tr}_{2}(M)$ is layered, then each subset (cf definition 11) of $T$ has the property that no two elements in it are instances of events in $\Sigma_{R j}$ for any $R j \in M$.

Proof: Any two elements $i l, i 2$ (corresponding to events $e l, e 2$ ) in the same subsel of $\%$ must be concurrent (definitions 3.11). Suppose el.e2 $\in \Sigma_{\mathrm{Rj}}$ with $\mathrm{Rj} \in \mathrm{M}$. Then $\left.\mathrm{I}\right|_{\Sigma_{\mathrm{Rj}}}$ will include $i l, i 2$ which will be concurrent (definition 2). Hence $\left.r\right|_{\Sigma_{R j}}$ cannot be a total order and therefore $\mathrm{T} \mathrm{Tr}_{2}(\mathrm{M})$ (definition 4$) \cdots$ leading to a contradiction. Hence the result.

\section{Theorem 12}

Proof: The behavior we require of the external world is that it simultancously raise REQ for all events in the first subset of $T$, wait until all corresponding ACK are high, then simultaneously lower all REQ, wait until all ACK are low, then repeat this cycle for the next subset of $\mathrm{T}$, and so on. We need to show that under these conditions the circuit responds within a finite amount of time in each cycle. The result then follows directly.

As shown in the proof of Jemma 20, all ACK's are initially low. Hence they are low at the beginning of each of the cycles mentioned in the previous paragraph. At the beginning of each such cycle, Ext.Int and every $\operatorname{Seq}(j)$ with $R j \in M$, get redefined. Let 'Tp denote 'I restricted to subsets before the current cycle. It is casy to show by induction on the number of cycles and definition 4 that at the beginning of each cycle $T(E x t)=T p$ and $T p \in$ $\operatorname{Tr}_{\Sigma}(M)$. Hence for any $R j \in M, S\left(\left.T P\right|_{\Sigma_{R j}}\right)$ is a prefix of some element in $\mathrm{L}_{\mathrm{Rj}}$. If the next subset contains an instance $i l$ of event el, then for each $R j \in M$ such that $e l \in \Sigma_{R j}, S\left(\left.T p\right|_{\Sigma_{R j}}\right.$ ) can be extended by $i$ to give a prefix of some sequence in $L_{R j}$; in fact this extension gives the next value of $\left.T \mathrm{p}\right|_{\Sigma_{\mathrm{p}}}$ (see lemma 22). But by leminas 18,17, fur any $R j \in M, T(\operatorname{Seq}(j))=\left.T(E x t)\right|_{\Sigma_{R j}}=$ $\left.T p\right|_{\Sigma_{R j}}$. Hence for each $R j \in M$, such that el $\in \Sigma_{R j}, T(\operatorname{Seg}(j))$ can be extended by il to give a prefix of some sequence in $\mathrm{L}_{\mathrm{Rj}}$. Thus by proposition 8 , the corresponding sequencers SEQ , with $e l \in \mathbf{\Sigma}_{\mathrm{Rj}}$ will have Isss ${ }_{j}$ low. This applies to any $e l$ in the next subset of $T$.

Thercfore at the beginning of any cycle, when REQ $_{e l}$ for any event $e l$ in the next subset of $T$ is raised, all DIS $l$ inputs to the NOR gate for cvent el (sce Figure 3-2). will be low. Nlso within a finite amount of time all relevant $\mathrm{r} \Lambda_{e l}$ 's must go low by proposition 8 , since the corresponding $7 \mathrm{TR}$ el s are already low. Hence $\mathrm{CLR}$ el will go low. and $I N_{e l}$ will go high for cach $e l$ in the next subset of T. It follows from proposition 6 and lemma 22 that all ACK's corresponding to crents in the next subsel of 'l' will be raised within a finitc amount of time.

The proof for the second half of the cycle is morc straightforward. By lemma 8 once all REQ's are lowered, within a finite time all relevant 'TA's will be raiscd, causing the corresponding CLR's to 80 high. As a result all relevant IN's go low (sec figure 3-2) and hence by proposition 6 all ACK's go low within a finite timc, completing the cycle. 